
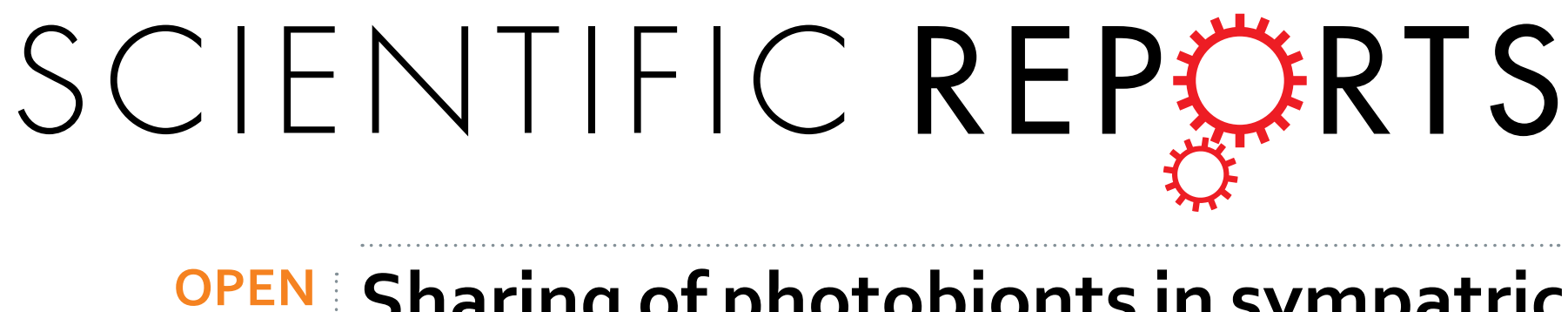

\title{
Sharing of photobionts in sympatric populations of Thamnolia and Cetraria lichens: evidence from
}

Received: 19 July 2017

Accepted: 1 February 2018

Published online: 13 March 2018

\section{high-throughput sequencing}

loana Onuț-Brännström¹, Mitchell Benjamin ${ }^{1}$, Douglas G. Scofield $\mathbb{D}^{2,3}$, Starri Heiðmarsson ${ }^{4}$, Martin G. I. Andersson ${ }^{5}$, Eva S. Lindström $\mathbb{1 D}^{5}$ \& Hanna Johannesson ${ }^{1}$

In this study, we explored the diversity of green algal symbionts (photobionts) in sympatric populations of the cosmopolitan lichen-forming fungi Thamnolia and Cetraria. We sequenced with both Sanger and Ion Torrent High-Throughput Sequencing technologies the photobiont ITS-region of 30 lichen thalli from two islands: Iceland and Öland. While Sanger recovered just one photobiont genotype from each thallus, the lon Torrent data recovered 10-18 OTUs for each pool of 5 lichen thalli, suggesting that individual lichens can contain heterogeneous photobiont populations. Both methods showed evidence for photobiont sharing between Thamnolia and Cetraria on Iceland. In contrast, our data suggest that on Öland the two mycobionts associate with distinct photobiont communities, with few shared OTUs revealed by lon Torrent sequencing. Furthermore, by comparing our sequences with public data, we identified closely related photobionts from geographically distant localities. Taken together, we suggest that the photobiont composition in Thamnolia and Cetraria results from both photobiont-mycobiont codispersal and local acquisition during mycobiont establishment and/or lichen growth. We hypothesize that this is a successful strategy for lichens to be flexible in the use of the most adapted photobiont for the environment.

Symbiosis is any type of close and long-term biological interaction between at least two different organisms. Across the Tree of Life, there are numerous examples of independently arisen symbiotic interactions with similar ecological and/or morphological outcomes ${ }^{1}$ among which lichens and reef corals being representative examples. These long-lived symbiotic entities are formed by heterotrophic organisms, fungi or animals, which acquire their carbon from the autotrophic microbes. Lichens, in contrast to the climate dependent corals ${ }^{2}$, are successful colonizers of moderate to extreme biomes and climatic zones ${ }^{3}$ and outperform vascular plants in both biodiversity and biomass in these areas ${ }^{4}$. Still, the slow growth of lichens and the impracticability of experimental work have resulted in a largely unknown life cycle and a limited understanding of the mechanism of adaptation to the environment. The focus of this study is the dynamics of the association between the heterotrophic fungus (i.e., the mycobiont) and the autotrophic microbe (the photobiont) that constitute lichens.

It is widely accepted that the mycobiont, with its net-like mycelium, provides the lichen its shape and structure, while the photobiont contributes with the carbon source ${ }^{5}$. The mycobiont also offers the matrix of the symbiotic vegetative propagules (soredia, isidia or minute thallus fragments), where symbiotic partners are embedded and dispersed as one functional unit to form a new lichen individual ${ }^{6,7}$. In addition, many lichenized fungi reproduce sexually by producing meiospores that can disperse and form new lichens by acquiring compatible photobionts and other symbiotic partners from external sources ${ }^{5}$. The fact that the most common genera of green algae photobionts (Trebouxia and Pseudotrebouxia) rarely form free-living colonies as their aerial relatives ${ }^{7}$ ), make lichens' source of new photobionts puzzling ${ }^{8}$. It has been hypothesized that free-living mycobionts are ephemeral,

${ }^{1}$ Systematic Biology, Department of Organismal Biology, Uppsala University, Uppsala, Sweden. ${ }^{2}$ Evolutionary Biology, Department of Ecology and Genetics, Uppsala University, Uppsala, Sweden. ${ }^{3}$ Uppsala Multidisciplinary Center for Advanced Computational Science (UPPMAX), Uppsala University, Uppsala, Sweden. ${ }^{4}$ Icelandic Institute of Natural History, Akureyri Division, Borgir Nordurslod, Iceland. ${ }^{5}$ Limnology, Department of Ecology and Genetics, Uppsala University, Uppsala, Sweden. Correspondence and requests for materials should be addressed to H.J. (email: hanna.johannesson@ebc.uu.se) 
and an advantageous strategy for lichens that disperse by meiospores is to use as immediate source photobiont cells from already established lichens, dying lichens or vegetative propagules ${ }^{9,10}$. Accordingly, lichen lineages do not constitute cospeciating symbiotic fungi and algae; rather, horizontal transmission (i.e., switching) of photobionts between different fungal lineages frequently occurs ${ }^{11,12}$. Photobiont switching seems to be an ubiquitous phenomenon in lichens and appears to play a vital role in lichens adaptation to environment ${ }^{8,13}$. Furthermore, this is not a lichen specific phenomenon, but was also shown in reef corals, which can survive elevate temperatures and recover from bleaching episodes if they succeed to associate with a specifically adapted photobiont lineage $^{2,14}$. Although the photobiont adaptive hypothesis was only occasionally experimentally tested in lichens ${ }^{15,16}$, several studies have shown a strong correlation between the photobiont genotype and ecology or geography of the lichen ${ }^{4,17,18}$. Additionally, it was shown that photobiont genotypes can be shared by diverse mycobionts at the same location ${ }^{17,19-30}$. All of these findings advocate that lichen forming fungi associate with local adapted photobiont strains that will be selected and hence increase in frequency within population ${ }^{19}$. Lichens photobionts do not show a significant population structure between localities, but identical photobiont genotypes were shown to be encountered across wide geographic ranges ${ }^{4,31}$, suggesting that the vegetative dispersing mode of lichens is an important contributor on photobiont distribution ${ }^{23,32}$.

At the same time, it is yet not clarified how many photobiont genotypes individual lichen thalli can contain. Traditional Sanger sequencing of PCR products containing amplicons of ITS-region has been used for the molecular identification of photobionts in lichen thalli (e.g. ${ }^{31}$ ) and this methods is not optimal for detecting multiple photobionts in a tissue. However, the results from studies using different methodological approaches, including High-Throughput Sequencing (HTS), challenge the dogma of single photobiont genotypes in a lichen thallus, and suggest the presence of a heterogeneous photobiont population within a lichen individual ${ }^{15,33-37}$.

The aims of this study were (1) to explore the diversity of photobionts in lichen symbioses in nature, and (2) to compare the sensitivity of Sanger and IonTorrent HTS technologies for detecting photobiont genotypes in lichens. For this purpose, we used a simple study design to investigate photobionts of two cosmopolitan, ecological similar but distantly related ${ }^{38}$ lichenized fungal genera, Thamnolia Ach. ex Schaer. and Cetraria Ach. We investigated the diversity of photobionts in sympatric populations of Thamnolia and Cetraria from two geographic localities (Iceland and Öland). We also investigated whether the retrieved local photobiont genotypes can be encountered in Thamnolia and Cetraria individuals from other parts of the world. Our HTS data suggest that multiple photobiont genotypes coexist within lichen thalli and revealed sharing of photobionts in sympatric population. This study increases our understanding of the nature of symbioses formed by heterotrophs and autotrophs, and may spur other researchers to use HTS methodology to examine the photobiont community of lichens.

\section{Materials and Methods}

Study system. Thamnolia and Cetraria were selected since they are often found growing together in nature. Furthermore, there are previous reports of symbiont flexibility in Thamnolia ${ }^{31,39}$ and photobiont switching has previously been correlated with the colonization of climatically different regions in Cetraria ${ }^{4}$. Both Thamnolia and Cetraria can be encountered in high alpine and tundra environments, but Cetraria species have a wider distribution and are also found in the boreal forest or even warm, arid habitats ${ }^{4}$. Both genera appear to mainly reproduce through vegetative propagules, via lichen fragments as in Thamnolia ${ }^{40-42}$ or through diaspores (isidia: small outgrowths of lichen thallus) produced by Cetraria ${ }^{4}$. In addition, both genera produce fungal spores that will need to re-associate with local photobionts to form new lichens ${ }^{4,43}$.

Field sampling and morphological identification. The lichen samples used for this study were collected from two islands representing two different habitats: the melur of Iceland (sandy, gravelly hills with sparse vegetation $)^{44}$ and the alvar of Öland, Sweden (limestone plain with very thin soil layer $)^{45}$. On Iceland one site was sampled (Gardabaer), while on Öland two sites were sampled (Öland 1 and Öland 2) (Fig. 1, Table 1). There was a noticeable difference in the soil color at the different alvar sites; while Öland 1 had a yellowish color, indicating goethite enrichment and an increased moisture availability, Öland 2 had a reddish color, which indicates a good soil drainage and the presence of unhydrated iron oxides ${ }^{46}$. The field sampling was performed as follows: within an area of $400 \mathrm{~m}^{2}$, five circles with one-meter radius and with at least two meters distance between each other were selected (Fig. 1). Within each circle, Thamnolia and Cetraria specimens (individual lichen thalli) were collected and identified as Thamnolia subuliformis (Ehrh.) W.L. Culb., Cetraria aculeata sensu lato (Schreb.) Fr. or Cetraria islandica (L.) Ach by using morphological characters. For further analyses, we used one specimen of each genus from each circle (Fig. 1, Table 1).

DNA extraction. For each selected specimen, we divided the lichen tissue in two halves: one half to be analysed using Sanger sequencing and one using HTS based Ion Torrent technology (see Supplementary Fig. S1). For Sanger sequencing, DNA extractions were separately performed on all 30 specimens listed in Table 1 . For Ion Torrent sequencing we pooled for each locality the five specimens of Thamnolia into one sample, and the five specimens of Cetraria in another sample (Table 1, see Supplementary Fig. S1). We used $5 \mathrm{mg}$ tissue of each specimen for DNA extraction. For both sequencing technologies, DNA was isolated with DNeasy Plant Mini Kit (Qiagen, GmbH, Hilden, Germany).

Sanger sequencing of the mycobiont and photobiont ITS regions. PCR reaction was executed with the Thermo-Scientific Green Phusion High-Fidelity DNA polymerase kit (Thermo Fisher Scientific Inc. Waltham, MA, USA), according to the manufacturer's instructions. From each of the 30 samples of Table 1, the ITS region of the mycobiont was amplified with the general primers ITS1F and ITS4, and the ITS region of the photobiont was amplified using the general primer pair ITS1T and ITS4T (Table S1). The PCR products were cleaned of unwanted dNTPs and primers using Exonuclease I (Thermo Fisher Scientific, SE) and Shrimp Alkaline 

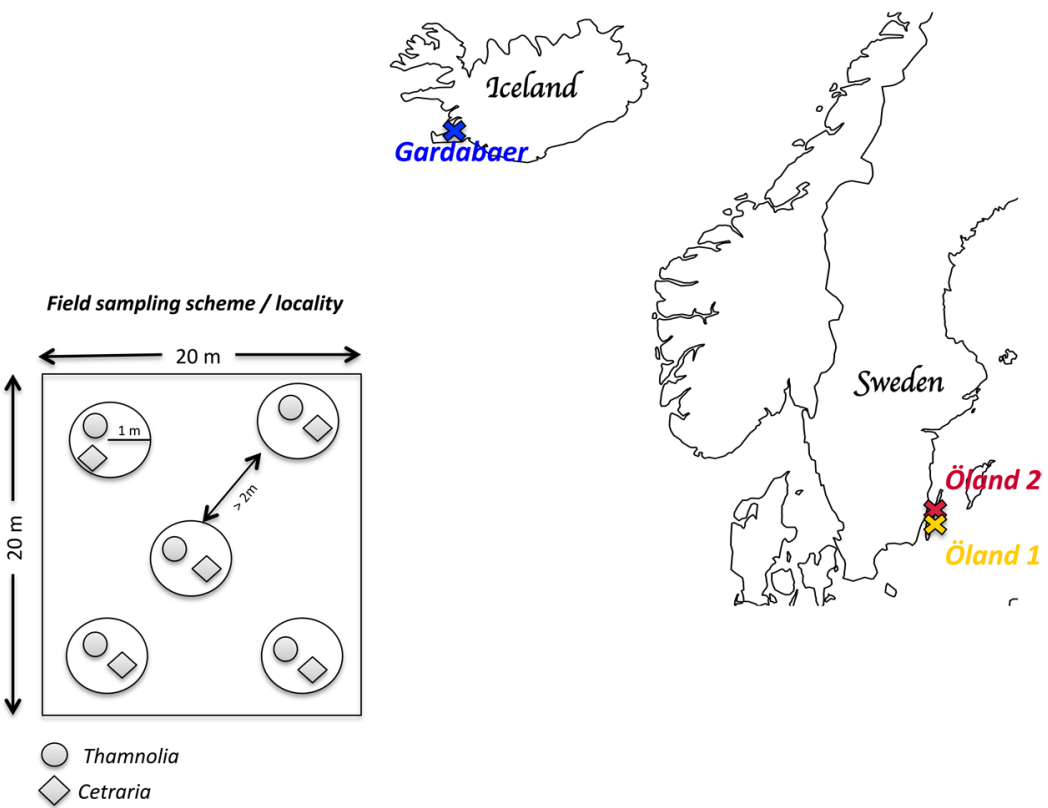

Figure 1. Investigated localities and sampling scheme. The map with the three investigated sites: one on Iceland (Gardabaer) and two in Sweden (Öland 1 and Öland 2) and the field sampling protocol. Within an area of $400 \mathrm{~m}^{2}$, five circles with one-meter in radius and with at least two meters distance between each other were selected. Within each circle, Thamnolia and Cetraria specimens (individual lichen thalli) were collected and for further analyses, we used one specimen of each genus from each circle.

Phosphatase (Affymetrix). The BigDye Teminator v 3.1 Cycle Sequencing and BigDye XTerminator Purification Kits (Thermo Fisher Scientific Inc. Waltham, MA, USA) were used to prepare the sample to be sequenced with Sanger technology by the ABI 3730 XL machine of Evolutionary Biology Centre, Uppsala University. All PCR products were sequenced from both directions using the same primers that were used for PCR amplification. The chromatograms of the sequences were visually inspected and curated by using the program Sequencher v5.2.3 (Gene Codes Corporation, Ann Arbor, MI, USA) and Geneious R9 (http://www.geneious.com) ${ }^{47}$. Sequencher was used to identify heterozygous sites in the sequences generated by Sanger technology: when two peaks with over $80 \%$ area overlap were encountered in a sequence, the site was identified as heterozygous, and the IUPAC ambiguity code was used for that particular site.

Verification of mycobionts in the pooled samples. To verify that we did not have any cross-contamination between samples of Thamnolia and Cetraria in the six pooled samples of lichens used for Ion Torrent sequencing (Table 1), we used genus specific primers to verify the mycobiont. As control, we mixed DNA from the two genera in ratios of 1:9, 1:5 and 1:1 and performed two rounds of amplification on all of these DNA samples by using either Thamnolia and Cetraria fungal ITS specific primers designed for this study (Table S1).

Ion Torrent sequencing of the ITS1 region of photobionts. PCR was executed with the Thermo-Scientific Phusion High-Fidelity DNA polymerase kit to amplify a part of the algal ITS1 region of maximum size $350 \mathrm{pb}$, using barcoded primers specifically designed for this study by the bioinformatics support from the Ion Torrent Sequencing center at SciLifeLab in Uppsala (Table S1). We also used two negative controls in which the template DNA was replaced by sterile water. Each sample for PCR (including the negative control) had its own barcoded primer combination. Gel electrophoresis was used to confirm the presence of PCR products of the expected size in the samples with DNA and the absence of PCR products in the negative controls. We used DNA Clean and Concentrator kit (Zymo Research, Orange, CA) to clean the amplicons from unwanted primers and dNTPs. The PCR product concentrations were measured with Qubit fluorometric quantification (Life Technologies), where after a single solution with all barcoded amplicons represented in equal amounts, and the two negative controls, was created. The library preparations with fusion primers were performed according to the manufacturers instructions. Uppsala SciLifeLab sequenced the pooled amplicons samples on an Ion Torrent PGM platform (Ion Torrent Xpress) using the $314^{\mathrm{TM}}$ Chip KIT and $400 \mathrm{bp}$ reads. The raw reads are deposited to the European Nucleotide Archive (ENA) (see Table 1 for the ENA accession numbers).

Delineating and quantifying operational taxonomic units from lon Torrent data. The Ion Torrent reads were processed to trim adapter and low-quality sequences using TorrentSuite BaseCaller 4.0-6/76303. To verify adapter removal, we constructed an adapter sequence list using Ion Torrent documentation, choosing A and P1 adapters along with the common prefix of barcode adapters, and scanned FastQ-format read sequences for each sample using FastQC (http://www.bioinformatics.babraham.ac.uk/projects/fastqc/). None of the candidate adapter sequences were detected. 


\begin{tabular}{|c|c|c|c|c|c|c|c|c|c|}
\hline \multirow[b]{2}{*}{ Site } & \multirow[b]{2}{*}{ habitat } & \multirow[b]{2}{*}{$\begin{array}{l}\text { Geographic } \\
\text { description }\end{array}$} & \multirow[b]{2}{*}{ Lichen species } & \multirow[b]{2}{*}{ Individual sample ID } & \multirow[b]{2}{*}{$\begin{array}{l}\text { Pool } \\
\text { ID }\end{array}$} & \multirow{2}{*}{$\begin{array}{l}\text { ENA } \\
\text { accession } \\
\text { number }\end{array}$} & \multicolumn{3}{|c|}{ GenBank Sanger ITS } \\
\hline & & & & & & & $\begin{array}{l}\text { herbarium } \\
\text { voucher }\end{array}$ & mycobiont & photobiont \\
\hline \multirow{8}{*}{ Iceland } & \multirow{8}{*}{ melur } & \multirow{8}{*}{$\begin{array}{l}\text { Iceland, Vestur- } \\
\text { Island, Garðabær } \\
\text { municipality, S of } \\
\text { Vifilsstaðaháls }\end{array}$} & Thamnolia subuliformis & T.sub1_Ice & \multirow{4}{*}{1} & \multirow{4}{*}{ ERR2307093 } & L-851027 & MG250331 & MG250287 \\
\hline & & & Thamnolia subuliformis & T.sub3_Ice & & & L-851029 & MG250333 & MG250289 \\
\hline & & & Thamnolia subuliformis & T.sub4_Ice & & & L-851030 & MG250334 & MG250290 \\
\hline & & & Thamnolia subuliformis & T.sub5_Ice & & & L-851031 & MG250335 & MG250291 \\
\hline & & & Cetraria aculeata s. lat & C.acu2_Ice & \multirow{4}{*}{2} & \multirow{4}{*}{ ERR2307094 } & L-851035 & MG250318 & MG250303 \\
\hline & & & Cetraria aculeata s. lat & C.acu3_Ice & & & \begin{tabular}{|l|} 
L-851036 \\
\end{tabular} & MG250319 & MG250304 \\
\hline & & & Cetraria islandica & C.isl4_Ice & & & L-851032 & MG250320 & MG250305 \\
\hline & & & Cetraria aculeata s. lat & C.acu5_Ice & & & L-851037 & MG250321 & MG250306 \\
\hline \multirow{6}{*}{ Öland 1} & \multirow{6}{*}{ alvar } & \multirow{6}{*}{$\begin{array}{l}\text { Sweden, Öland Island, } \\
\text { Stora Alvaret, between } \\
\text { S. Bårby and Alby }\end{array}$} & Thamnolia subuliformis & T.sub6_Oel1 & 3 & ERR2307095 & L-851013 & MG250336 & MG250292 \\
\hline & & & Cetraria aculeata s. lat & C.acu6_Oel1 & \multirow{5}{*}{4} & \multirow{5}{*}{ ERR2307096 } & L-851017 & MG250322 & MG250307 \\
\hline & & & Cetraria islandica & C.isl7_Oel1 & & & L-851022 & MG250323 & MG250308 \\
\hline & & & Cetraria islandica & C.isl8_Oel1 & & & L-851018 & MG250324 & MG250309 \\
\hline & & & Cetraria aculeata s. lat & C.acu9_Oel1 & & & L-851019 & MG250325 & MG250310 \\
\hline & & & Cetraria aculeata s. lat & C.acu10_Oel1 & & & L-851020 & MG250326 & MG250311 \\
\hline \multirow{5}{*}{ Öland 2} & \multirow{5}{*}{ alvar } & \multirow{5}{*}{$\begin{array}{l}\text { Sweden, Öland } \\
\text { Island, Stora Alvaret, } \\
\text { between S. Möckleby } \\
\text { and Torngård }\end{array}$} & Thamnolia subuliformis & T.sub11_Oel2 & \multirow{5}{*}{5} & \multirow{5}{*}{ ERR2307097 } & L-851023 & MG250341 & MG250297 \\
\hline & & & Thamnolia subuliformis & T.sub12_Oel2 & & & L-851008 & MG250342 & MG250298 \\
\hline & & & Thamnolia subuliformis & T.sub13_Oel2 & & & L-851009 & MG250343 & MG250299 \\
\hline & & & Thamnolia subuliformis & T.sub14_Oel2 & & & L-851011 & MG250344 & MG250300 \\
\hline & & & Thamnolia subuliformis & \begin{tabular}{|l|} 
T.sub15_Oel2 \\
\end{tabular} & & & L-851010 & MG250345 & MG250301 \\
\hline
\end{tabular}

Table 1. Lichen samples used in this study. All samples were deposited to The Museum of Evolution Herbarium, Uppsala University, Sweden (herbarium vouchers). GenBank accession numbers for the ITS sequences amplified through Sanger sequencing from the mycobiont and photobiont are given for each sample. For each locality lichen tissue from all five samples from one genus (Thamnolia or Cetraria) were pooled together for the Ion Torrent sequencing (pooled samples). ENA accession numbers for the amplicons generated by IonTorrent are shown for each pool. *Unsuccessful PCR reaction.

To cluster amplicon sequences into operational taxonomic units (OTUs) for photobiont delineation and quantification, we used USEARCH 8.1.1861 ${ }^{48}$ following recommended practices for Roche 454 reads (http://drive5. com/usearch/manual/upp_454.html, accessed 2016/06/01), which have an error profile similar to data obtained by Ion Torrent sequencing. All OTU-related trimming, clustering and quantification steps were performed using USEARCH. We explored a variety of read trim lengths and expected-error counts using the -fastq_eestats2 option, and selected trimming to $200 \mathrm{bp}$ with 1.5 expected errors per read; this allowed us to retain sufficient length for each OTU to make comparisons with our Sanger sequences and sequences downloaded from databases. FastQ reads for all samples were pooled, dereplicated, and clustered into OTUs using default settings, with the additional constraint of requiring each OTU to be supported by at least two reads ( - minsize 2$)$. Thus, OTUs were formed using reads from all samples analysed together, with singleton OTUs excluded. Abundances of OTUs within each pooled set of reads were then estimated via global alignment of sampled-tagged reads, with minimum identity $97 \%$. This method ensures continuity of OTUs across samples, enables formation of OTUs that might only have sufficient read support in aggregate while avoiding formation of singleton OTUs, and allows for OTUs to be quantified within samples in which they are rare. The script implementing this pipeline together with the candidate adapter sequences used during the scan with FastQC are available at https://github. com/douglasgscofield/pubs/tree/master/Brannstrom-et-al-1.

To example the dependence of OTU richness estimates on read counts, which varied somewhat among samples (mean $36740 \pm 8300$ [s.d.], range 22486-43110), we determined rarefaction curves using estimated_observation_richness.py in QIIME 1.9.1 ${ }^{49,50}$. Richness estimates differed little throughout the range of observed read counts (see Supplementary Fig. S2), so we made no further correction to OTU diversity estimates. 
Taxonomic verification of mycobionts and photobionts. To assign the taxonomic identity of the mycobiont for each specimen identified as Thamnolia or Cetraria based on morphological characters, we compared the newly generated mycobiont and photobiont ITS sequences, both Sanger and Ion Torrent data, to sequences available at the GenBank nucleotide database ${ }^{51}$. We identified the best hits based on query coverage, sequence identity and e-value. The best hits that also had a species name assigned, using additional supporting evidence, were retrieved from GenBank. Subsequently, we employed a phylogenetic approach (see below) to investigate the relationship among the retrieved GenBank sequences and our newly generated data.

Phylogenetic analyses. For each independent analysis, the sequences were aligned with MAFFT ${ }^{52}$ and trimmed in AliView v1.18 ${ }^{53}$. All sequence alignments and the tree files resulted from this study analyses were deposited to https:/github.com/douglasgscofield/pubs/tree/master/Brannstrom-et-al-1.

We chose the most appropriate strategy for phylogenetic analyses based on the presence of heterozygous sites and the amount of variable sites within each alignment.

Since we identified heterozygous sites in the data from photobiont Sanger sequences of the complete ITS region, the program RRHS version 1.0.0.2 ${ }^{54}$ was used to randomly draw (with replacement) 1000 phased alignments of the trimmed alignment of the 30 photobiont Sanger sequences. For each of the 1000 phased alignments, phylogenetic trees were generated using the maximum likelihood (ML) with GARLI $2.01^{55}$ by running for each alignment five independent searches. Kimura 80 was selected as model for sequence evolution using the jModelTest. The 1000 trees with the best likelihood score were compiled in one phylogeny that represents the $50 \%$ majority-rule consensus tree using the program SumTrees ${ }^{56}$.

Thamnolia and Cetraria are highly divergent mycobiont genera from Lecanoromycetes ${ }^{38}$. Therefore we performed a separate phylogeny for samples of each genus based on the mycobiont Sanger sequences of the complete ITS region of our newly generated data together with GenBank references of sequences. For Thamnolia we chose representative ITS sequences for the previously discovered lineages A, B and $\mathrm{C}^{31}$ with the following accession numbers: KY550180, KY550083, KY550130, KY550098, KY550079, KY550170, KY550163, KY550143, KY550153, KY550127, KY550194. For Cetraria we used ITS sequences previously identified as C. aculeata, C. muricata and C. islandica (Accession numbers: AF228302, AF192409, EF373589, EF373567, AF228299). Both sets of sequences were aligned in AliView with MAFFT, and TrNef $+\mathrm{G}$ was chosen with JModel Test as sequence evolution model. Maximum likelihood phylogenies were performed with GARLI 2.01 together with 500 bootstrap trees. SumTrees script was used to place the bootstrap values on the branches.

The alignment of our newly generated photobiont sequences (ITS1 region) amplified with Sanger and Ion Torrent and the GenBank references (Table S2) presented many ambiguously aligned regions. To be able to use all sequence information, the software BAli-Phy $2.3 .8^{57}$, which simultaneously estimates the alignment and the phylogeny using Bayesian inference, was used. We used JModel test ${ }^{58}$ to define HKY with a gamma distribution as the nucleotide substitution model for the photobiont phylogeny. Five MCMC chains with 20000 iterations each were run. The convergence of chains was diagnosed with the program Tracer ${ }^{59}$ and with "bp-analyze.pl" script of BAli-Phy 2.3.8. The same script was used to calculate the posterior probabilities of the $50 \%$ majority-rule consensus tree of all the MCMC chains, after 2000 iterations were discarded as burn in. All phylogenies were visualized in FigTree 1.4.2 (http://tree.bio.ed.ac.uk/software/figtree/).

Sharing of photobiont OTUs. Non-metric multidimensional scaling (NMDS) was used to visualize similarities among the lichen samples with regard to photobiont diversity. The analyses were performed in $\mathrm{R}$ employing the function metaMDS in Vegan ${ }^{60}$ using the Bray-Curtis dissimilarity index. Before this analysis, the data sets were resampled (using the function rrarefy from Vegan) to an equal number of sequences per sample (22486). The NMDS analysis was run for 2 dimensions and 2000 iterations and the plot was generated in R using ggplot $2^{61}$. The script used for this analysis is available at https://github.com/douglasgscofield/pubs/tree/master/ Brannstrom-et-al-1.

The number of shared and specific OTUs between Thamnolia and Cetraria for each of the three localities was visualized as Venn diagrams that were constructed using the following web tool: http://bioinformatics.psb.ugent. be/webtools/Venn/.

The relative quantities of photobiont OTUs shared at each sampling location between Thamnolia and Cetraria were visualized by a hierarchical cluster analysis. The OTU read counts per sample were normalized to range from $0-1$ within each sample, and were then clustered by similarity separately among OTUs and among site/ genus pairs using hclust and dist in R. Distances were calculated via the Canberra method, as this permitted clustering to remain sensitive to similarities among more rare OTUs, unlike Euclidean distances, while retaining quantitative sensitivity unlike Boolean presence/absence distances. For display, OTU quantities were scaled by their cube-root, and colored by clade membership as determined by the phylogenetic analysis described above. The R script implementing this analysis is available at https:/github.com/douglasgscofield/pubs/tree/master/ Brannstrom-et-al-1.

Data Availability. All the herbarium vouchers are deposited in UPS (The Museum of Evolution Herbarium - Uppsala, Sweden) and their herbarium accessions numbers can be found in Table 1. All the DNA sequences are deposited in GenBank and ENA. The accession numbers can be found in Table 1 (for the Sanger sequences) and Table 2 (for the Ion Torrent sequences). DNA sequences alignments are uploaded online in https://github.com/ douglasgscofield/pubs/tree/master/Brannstrom-et-al-1.

The scripts used for the different analyses are deposited at https:/github.com/douglasgscofield/pubs/tree/ master/Brannstrom-et-al-1. 


\begin{tabular}{|c|c|c|c|c|c|c|c|c|}
\hline \multirow[b]{2}{*}{ OTUs ID } & \multicolumn{2}{|l|}{ Iceland } & \multicolumn{2}{|l|}{ Öland 1} & \multicolumn{2}{|l|}{ Öland 2} & \multirow[b]{2}{*}{ NC } & \multirow{2}{*}{$\begin{array}{l}\text { GenBank } \\
\text { numbers }\end{array}$} \\
\hline & Thamnolia & Cetraria & Thamnolia & Cetraria & Thamnolia & Cetraria & & \\
\hline OTU 1 & 38 & 9 & 41830 & 2 & 42020 & 0 & 1 & MG372066 \\
\hline OTU 2 & 520 & 41200 & 1 & 8974 & 0 & 4301 & 0 & MG372067 \\
\hline OTU 3 & 0 & 44 & 0 & 15220 & 2 & 33480 & 0 & MG372068 \\
\hline OTU 4 & 0 & 0 & 0 & 132 & 0 & 218 & 0 & MG372069 \\
\hline OTU 5 & 0 & 0 & 0 & 6209 & 0 & 0 & 0 & MG372070 \\
\hline OTU 6 & 0 & 0 & 0 & 47 & 0 & 115 & 0 & MG372071 \\
\hline OTU 7 & 0 & 0 & 0 & 393 & 0 & 821 & 0 & MG372072 \\
\hline OTU 8 & 0 & 0 & 0 & 12 & 0 & 16 & 0 & MG372073 \\
\hline OTU 9 & 0 & 0 & 75 & 0 & 61 & 0 & 0 & MG372074 \\
\hline OTU 10 & 0 & 0 & 24 & 0 & 28 & 0 & 0 & MG372075 \\
\hline OTU 11 & 0 & 0 & 0 & 11 & 0 & 27 & 0 & MG372076 \\
\hline OTU 12 & 1 & 0 & 108 & 0 & 158 & 0 & 0 & MG372077 \\
\hline OTU 13 & 4 & 346 & 0 & 68 & 0 & 43 & 0 & MG372078 \\
\hline OTU 14 & 0 & 0 & 0 & 8 & 0 & 0 & 0 & MG372079 \\
\hline OTU 15 & 0 & 0 & 9 & 0 & 13 & 0 & 0 & MG372080 \\
\hline OTU 16 & 0 & 0 & 5 & 0 & 3 & 0 & 0 & MG372081 \\
\hline OTU 17 & 124 & 2 & 0 & 3 & 0 & 0 & 0 & MG372082 \\
\hline OTU 18 & 23 & 5 & 0 & 0 & 0 & 1 & 0 & MG372083 \\
\hline OTU 19 & 0 & 0 & 5 & 0 & 12 & 0 & 0 & MG372084 \\
\hline OTU 20 & 404 & 1 & 0 & 2 & 0 & 0 & 0 & MG372085 \\
\hline OTU 21 & 0 & 0 & 0 & 1 & 0 & 3 & 0 & MG372086 \\
\hline OTU 22 & 0 & 0 & 7 & 0 & 3 & 0 & 0 & MG372087 \\
\hline OTU 23 & 10 & 11 & 0 & 6 & 0 & 1 & 0 & MG372088 \\
\hline OTU 24 & 21360 & 1 & 0 & 0 & 0 & 0 & 0 & MG372089 \\
\hline OTU 25 & 0 & 0 & 9 & 1 & 0 & 0 & 0 & MG372090 \\
\hline OTU 26 & 0 & 0 & 10 & 0 & 5 & 0 & 0 & MG372091 \\
\hline OTU 27 & 0 & 0 & 31 & 0 & 33 & 0 & 0 & MG372092 \\
\hline OTU 28 & 0 & 0 & 0 & 20 & 0 & 33 & 0 & MG372093 \\
\hline OTU 29 & 0 & 0 & 0 & 2 & 0 & 0 & 0 & MG372094 \\
\hline OTU 30 & 2 & 0 & 993 & 0 & 706 & 0 & 0 & MG372095 \\
\hline OTU 31 & 0 & 0 & 3 & 0 & 4 & 0 & 0 & MG372096 \\
\hline OTU 32 & 0 & 7 & 0 & 0 & 0 & 0 & 0 & MG372097 \\
\hline Total & 22486 & 41626 & 43110 & 31111 & 43048 & 39059 & 1 & \\
\hline
\end{tabular}

Table 2. Photobiont OTUs identified by Ion Torrent sequencing technology. The number of reads for each OTU in each pooled lichen and negative control (NC) sample is shown. For all samples the reads were pooled, dereplicated, and clustered into OTUs using the constraint of requiring each OTU to be supported by at least two reads ( - minsize 2$)$. A total number of reads obtained for each sample is summed up at the bottom of each column.

\section{Results}

DNA extractions, PCR, Sanger and lon Torrent sequencing. By using PCR and Sanger sequencing, we successfully amplified and sequenced the ITS region of the mycobiont from 29 of the 30 lichen specimens, and the ITS region of the photobiont from all 30. For the Ion Torrent sequencing of the photobionts, DNA was extracted and the ITS1 region was successfully amplified from all six pooled samples (Table 1). None of the negative controls showed amplicons by gel electrophoresis. Only one sequence of ITS per thallus was obtained by Sanger technology, while with Ion Torrent we discovered reads of multiple photobiont OTUs: after delineation and quantification of the photobiont amplicon sequences, 32 OTUs were identified. Each OTU ID together with the number of reads for all OTUs and their prevalence in each sample is shown in Table 2. Sequencing of the negative control resulted in 1 read (Table 2).

No cross-contamination of mycobionts in the pooled samples. PCR-reactions using Thamnolia or Cetraria specific primers (Table S1) verified that single genera were found in each pooled tissue-sample, and thus, that the samples were not cross-contaminated prior to the Ion Torrent sequencing step. Specifically, we amplified a PCR product from the Cetraria samples when Cetraria specific primers were used, but not when Thamnolia specific primers were used, and vice versa. In contrast, all samples that were mixed in different proportions with DNA originating from both Cetraria and Thamnolia tested positive with both primer combinations, which suggests that any cross contamination at the level of $10 \%$ would have been detected (data not shown). 
a. Thamnolia ML phylogeny

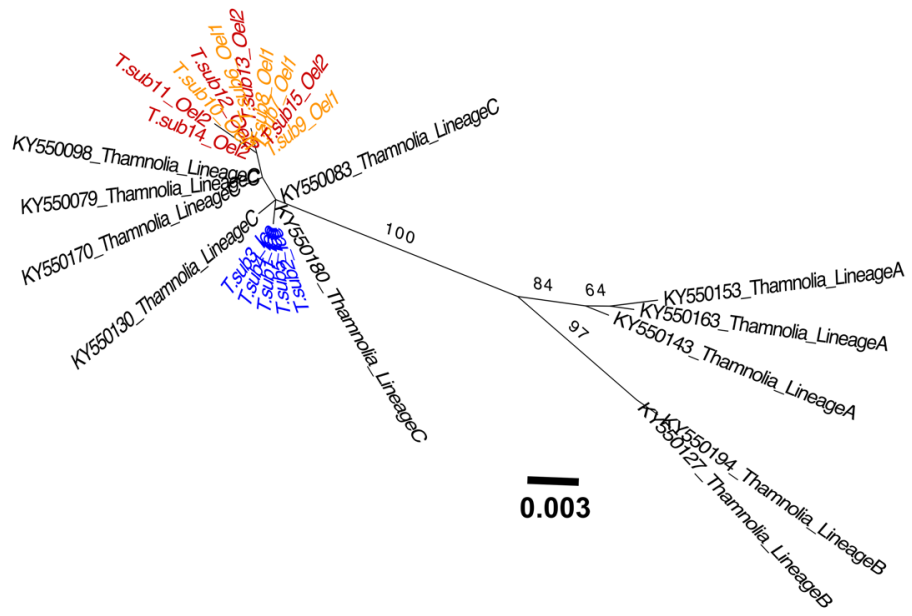

b. Cetraria ML phylogeny

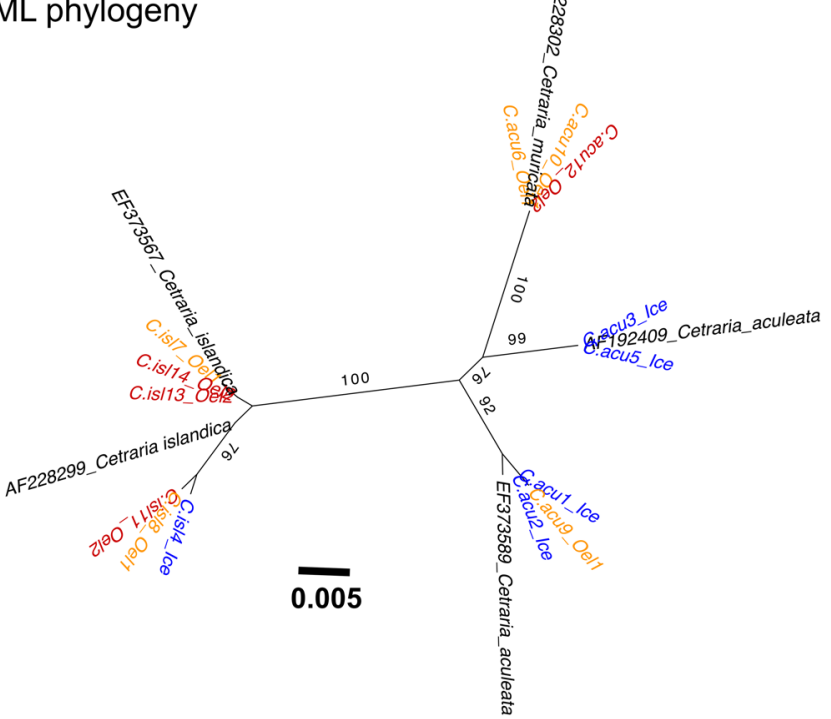

Figure 2. Unrooted mycobiont phylogenies of the Thamnolia (a) and Cetraria (b) mycobionts based on the complete ITS region obtained with Sanger sequencing. Our newly generated sequences are highlighted in colours: the samples from Iceland (Ice) in blue; the ones from Öland 1 (Oel1) in yellow and the ones from Öland 2 (Oel2) in red. 'C.acu' codes for Cetraria aculeata; 'C.isl' codes for Cetraria islandica, and 'T.sub' for Thamnolia subuliformis. Numbers from 1 to 15 are given for each Thamnolia and Cetraria specimen investigated in this study (see Table 1). The accession numbers and the species/lineage name of the sequences retrieved from GenBank are shown in black. The support $(<60 \%)$ for each branch is given as bootstrap values shown above branches. The scale bar indicates the branch length.

Taxonomic identification of the mycobiont using Sanger ITS data. The mycobiont phylogeny based on the Sanger sequences of the ITS region of Thamnolia (Fig. 2a) shows that all newly generated sequences group together with Thamnolia GenBank sequences of a clade previously identified as Thamnolia Lineage $\mathrm{C}^{31}$ and subsequently named Thamnolia subuliformis s. str. ${ }^{62}$. In contrast, our Cetraria phylogeny (Fig. 2b) splits our samples into four supported lineages. Based on the GenBank references sequences, shown in black font in Fig. 2b, all samples identified as $C$. islandica group together with the reference of $C$. islandica. However, the specimens that were morphologically identified as C. aculeata are paraphyletic, two groups cluster with C. aculeata reference and one with the one of C. muricata (Fig. 2b).

Taxonomic identification of the photobiont using Sanger and lon Torrent ITS data. In the phylogenetic analyses of the Sanger and Ion Torrent sequences of the ITS region of the photobionts, all 5 MCMC chains converged and were combined in the 50\% majority consensus rule tree. The Trebouxia impressa clade was 
used as outgroup. The phylogeny showed that both Thamnolia and Cetraria mycobionts are associated with several green algae of Trebouxia. Specifically, the phylogeny shows 7 well-supported main lineages of Trebouxia with posterior probabilities (PP) above $80 \%$. Because of the uncertain species status in Trebouxia, the 7 lineages are referred to herein as 'T. simplex A' 'T. simplex 2', T. simplex $C$ ' (further on divided in three other clades: 'C1', and 'C2'), 'T. angustilobata', 'T. simplex jamesii-vulpinae, 'T. gigantea-vagua', and 'T. impressa' (Fig. 3).

Most of the Ion Torrent sequences were found in four of the clades: 'T. simplex 2', 'T. simplex C1', 'T. simplex 1 ', and 'T. jamesii-vulpinae'. The exceptions were OTU 29 and OTU 14 which groups in the 'T. gigantean-vagua' clade together with a photobiont sequence amplified from a Thamnolia lichen specimen from China (accession number EU715061: Fig. 3). Notably, this sequence was amplified with Sanger technology by another research group and retrieved by us from GenBank.

Some of the photobiont clades were dominated by sequences retrieved from either Thamnolia or Cetraria. For example, 'T. impressa' and 'T. simplex 2' clades are dominated by Thamnolia's photobionts, while T. angustilobata', 'T. simplex C2', and 'T. jamesii-vulpinae' exclusively contain photobionts of Cetraria (Fig. 3). On the other hand, the subclade named ' $T$. simplex 1 ' contains photobiont sequences from both lichen genera, from all three investigated localities (Gardabaer, Öland 1 and Öland 2), but also from other localities of both hemispheres (Fig. 3).

Due to its divergence from the other sequences, OTU 25 was not included in the phylogeny and was identified based on a nucleotide blast search in the NCBI database. The best hit was Hemichloris antarctica which had a $100 \%$ query cover, an e-value of 1e-37 and an identity score of $80 \%$ was (GenBank accession number: HG972970). OTU 25 was found in low abundance in Cetraria (Öland 1, with 1 read) and Thamnolia (Öland 1, with 9 reads) (Table 2).

Sharing of photobionts between Thamnolia and Cetraria. Both the Sanger and the Ion Torrent datasets indicate that the photobiont community is more similar between Thamnolia and Cetraria on Iceland than on Öland. First, the photobiont phylogenetic analyses of the complete ITS region obtained with Sanger technology (see Supplementary Fig. S3) showed that the photobionts of Thamnolia and Cetraria from Iceland associate with closely related photobionts. Most of the photobionts of Cetraria specimens from Öland are closely related with the lichens from Iceland, while the Thamnolia photobionts form a separate clade (see Supplementary Fig. S3). The data from Ion Torrent shows the same trend but at higher resolution, since more genotypes were recovered by this analysis. Both clustering analyses of the Ion Torrent data (Figs 4 and 5) together with the phylogenetic analyses of the amplicons of the ITS1 region (Fig. 3) group the photobiont communities of the two lichen genera from Iceland together. The Venn diagrams of Fig. $5 \mathrm{~b}$ show that circa $67 \%$ of the photobiont OTUs are common between Thamnolia and Cetraria at melur (Iceland). Most of this shared photobiont OTUs belong to a clade identified as Trebouxia simplex 1 (Fig. 5a), which was also encountered in Cetraria specimens from Öland. The Icelandic Thamnolia and Cetraria were also found associated with a low frequency OTU (OTU 1) that was identified as Trebouxia simplex 2 (Fig. 5a). Noteworthy, OTU1 was found in high abundance in Thamnolia samples from Öland (Fig. 5a; Table 2).

At alvar (Öland 1 and Öland 2), each lichen genera have a high proportion of exclusive OTUs but also few shared ones (ca. 16\% for Öland 1 and 5\% for Öland 2) (Fig. 5b). Both Thamnolia pooled samples from the alvar are predominantly associating with OTUs genotyped as 'Trebouxia simplex 2' (Fig. 5a; Table 2). But they also contain two low frequencies OTUs (OTU2 and OTU3) that are found in high proportions in Cetraria lichens and they belong to 'Trebouxia simplex 1' and 'Trebouxia simplex C1', respectively. Cetraria samples from Öland1 were additionally associated with photobiont OTUs belonging to the genotypes of 'T. jamesii-vulpinae' (OTU 5), 'T. gigantean-vagua' (OTU14 and OTU29) (Fig. 5a,b) but also 'Trebouxia simplex 2' (OTU 1).

These overall results of differential sharing hold when applying more stringent filtering in requiring each OTU to be supported by at least 3, instead of 2 reads (see Supplementary Figs S4 and S5).

\section{Discussion}

Thamnolia and Cetraria associate with diverse, and partly overlapping, Trebouxia photo-

bionts. Our results confirm the findings of previous studies indicating that mycobionts of Thamnolia and Cetraria are flexible towards their photobionts in that they can associate with different Trebouxia lineages ${ }^{4,31,39}$ (Fig. 3). Although three of these photobiont lineages were detected only in either Thamnolia (Trebouxia 'impressa', Fig. 3) or Cetraria (Trebouxia 'jamesii-vulpinae', Trebouxia 'angustilobata', Fig. 3), all the other clades contained photobionts retrieved from both lichen genera. Our results were based on observations from three sites, but nevertheless showed that Thamnolia and Cetraria, which are often found growing together, can harbour an overlapping spectrum of genetically diverse photobionts in their thalli.

It is worth pointing out that we cannot with this study design confirm without doubt that the identified algal OTUs have their origin from within the thalli, and thus, that they represent true photobionts (c.f. refs ${ }^{36,63}$ ). Other studies, which were specifically interested in investigating the intrathalline photobiont diversity in several lichen species employed a more stringent approach by washing the lichen thalli with different solutions ${ }^{36,63}$. However, the only certain confirmation of the algal origin from within the thalli is the culturing of single cells of intrathalline photobionts ${ }^{33}$, or specific labelling, such as for example the one used in the recent study by Spribille et al. $2016^{64}$. Both of these last two approaches require a priori knowledge of the present intra-lichen diversity, which was not available at the onset of our study. The only taxon that appears to be epithallic from our data is OTU 25 , which is divergent (Hemichloris or similar, i.e., not a known lichen photobiont ${ }^{38}$ ) and was found in a small fraction of samples only on Öland 1, suggesting that external contamination is not a major issue for our study. Nevertheless, we are left with essentially the same result: sharing of a broad diversity of photobiont taxa, whether endo- or epithallic, which differs between species and sites. 


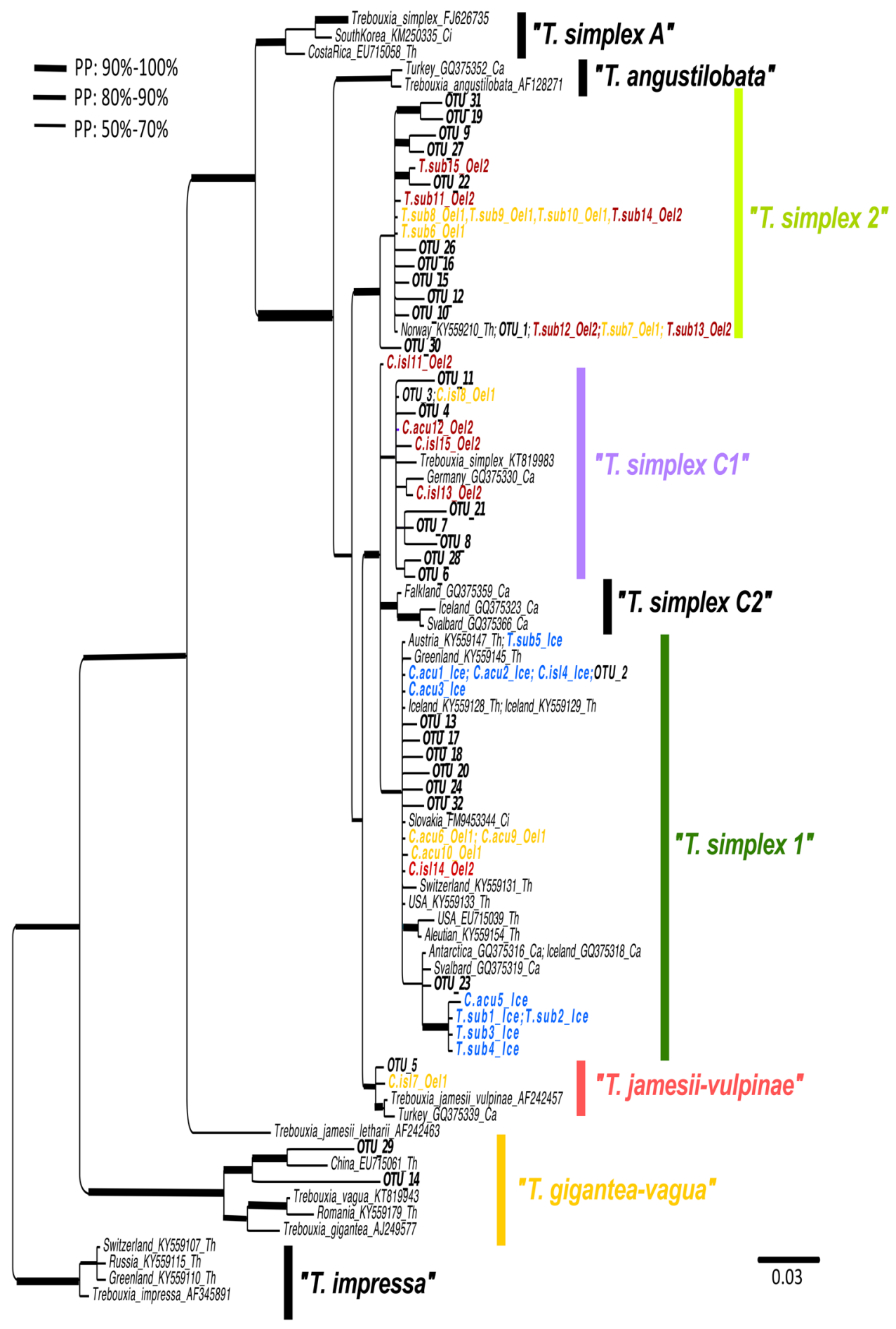

Figure 3. Rooted photobiont phylogeny based on the ITS 1 region, with data were obtained with Sanger and Ion Torrent sequencing technologies. Our newly generated Sanger sequences are highlighted in colors: the samples from Iceland (Ice) in blue; the ones from Öland 1 (Oel1) in yellow and the ones from Öland 2 (Oel2) are in red. 'C.acu' codes for Cetraria aculeata; 'C.isl' codes for Cetraria islandica, and 'T.sub' for Thamnolia subuliformis. Numbers from 1 to 15 are given for each Thamnolia and Cetraria specimen investigated in this study. The data obtained through Ion Torrent are in bold black and named as OTUs with their identity given as numbers from 1 to 32 . The GenBank numbers are shown in black; the accession numbers and the species/ lineage name can be seen in the sample ID. The support for each branch (posterior probabilities) is shown by branch thickness. The scale bar indicates the branch length.

HTS sequencing suggests that multiple photobiont genotypes exist within lichen thalli. The presence of a single photobiont in a lichen thallus usually found when using PCR and Sanger sequencing of molecular markers (e.g. ref. ${ }^{25}$ ) could be biologically true for certain lichens, but recent work indicates that it might 


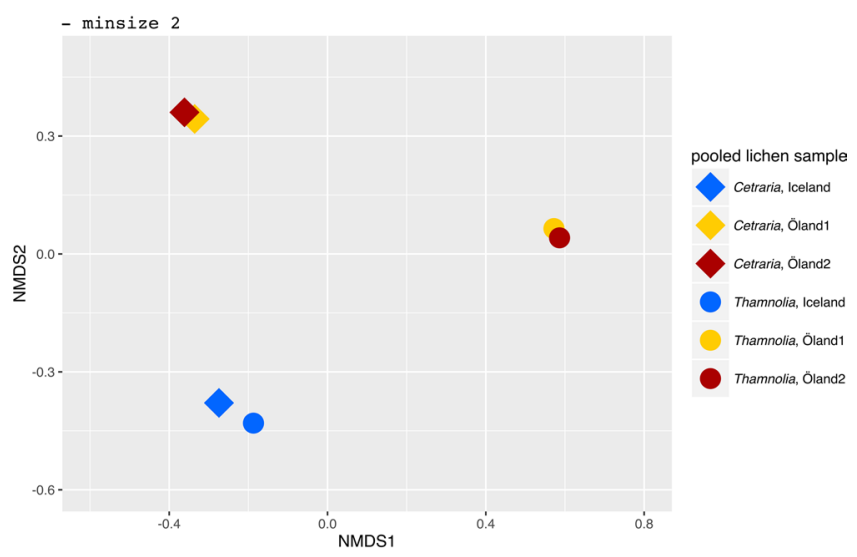

Figure 4. NMDS clustering of the photobiont composition, based on Ion Torrent data, of the six pooled lichen samples: one Thamnolia (depicted with circles) and one Cetraria (depicted with rhombs) from each of the three localities: Iceland (blue), Öland 1 (yellow) and Öland 2 (red). For all samples the reads were pooled, dereplicated, and clustered into OTUs using the constraint of requiring each OTU to be supported by at least two reads (-minsize 2). The Bray-Curtis dissimilarity was used and the stress value was 1 .

be an artefact of the traditional Sanger method, which is biased towards producing a predominant genotype as its single amplification product. Accordingly, with Sanger, we identified one genotype per individual thallus investigated herein, while the Ion Torrent data suggests the coexistence of several algal genotypes within single lichen individuals. Because our samples were pooled before Ion Torrent sequencing, we were not able to directly investigate the distribution of genotypes within individual thalli with this data. However, the finding of more photobiont genotypes (10 to 18 OTUs) than the number of studied thalli (5) in each sample suggests the simultaneous presence of multiple photobionts in Thamnolia and Cetraria lichens. The finding of more OTUs than number of thalli holds also when applying more stringent filtering in requiring each OTU to be supported by at least 3 reads, indicating that this result is not due to, e.g., rare OTUs originating from sequencing errors. Furthermore, we verified the coexistence of multiple photobiont genotypes within single lichen thalli by using specific primers to amplify and Sanger-sequence several Trebouxia lineages ('T. simplex', 'T. impressa' and 'T. vagua-gigantea' Fig. 3) (data not shown).

The idea that symbiotic organisms are not a homogenous population of autotrophic symbionts but rather a mosaic of local adapted photobiont strains was also shown in some corals systems ${ }^{65}$, and is consistent with several lichen studies that have used other molecular techniques such as restriction fragment polymorphism ${ }^{37}$, microsatellites $^{34}$ or HTS technologies to show that individual thalli contain more than one photobiont genotype $\mathrm{e}^{36,66}$.

In conclusion, all these recent studies are challenging the paradigm that lichen thalli contain homogeneous photobiont populations and highlight the importance of using more sensitive techniques than PCR and Sanger sequencing to the study of lichen photobionts. For example, if macrolichens such as Thamnolia and Cetraria contain heterogeneous intrathalline photobiont populations, approaches that reveal only the most abundant genotype (c.f. refs ${ }^{4,31,39,67}$ ) can give misleading results, e.g., infer photobiont switching and replacement in cases where instead dynamic fluctuations of photobiont within lichen thalli is taking place.

Photobiont sharing in sympatric populations of Thamnolia and Cetraria. Data from both sequencing technologies showed convincingly that the photobionts associated with Thamnolia and Cetraria from Iceland are closely related with each other, and with photobionts from Cetraria individuals from Öland (Figs 3 and 5a). Based on these genetic similarities we suggest that on Iceland, Thamnolia and Cetraria share photobionts, while on Öland the two lichen genera associate with distinct photobiont communities, although few OTUs were shared. Sharing of photobionts between closely related mycobiont species with similar ecologies was previously shown in lichens ${ }^{23,24}$ but rarely described between distantly related mycobiont genera ${ }^{66}$. Together with other studies, which show that lichen communities from harsh climatic conditions share photobionts ${ }^{20,25,66}$, our results support the hypothesis that mycobionts with similar ecological requirements, such as those specific to arctic environments, might share a common pool of photobionts ${ }^{21,12,17,35}$. Furthermore, the presence of similar genotypes across wide geographic ranges, combined with occurrence of multiple photobiont genotypes within lichen thalli, suggest a continuous exchange of photobionts between Thamnolia and Cetraria, by yet unknown mechanisms.

The significance of differential photobiont abundance in lichen thalli. If the proportion of reads generated by Ion Torrent sequencing is used to assess the predominant genotype within a lichen, we see that, as an example, on Öland one genotype (OTU3; T. simplex C1) is dominant in the Cetraria thalli, while on Iceland a genetically different photobiont is most abundant in Cetraria (OTU2; T. simplex 1) (Fig. 5a). Thamnolia sampled on Iceland also harbours OTUs belonging to T. simplex 1, but based on the proportion of reads shows more abundantly photobionts of OTU 24. The amount of the different photobiont genotypes in a lichen population may be a result of chance alone, or simply reflect the demographic history and/or the specificity of the mycobiont towards their photobionts ${ }^{68}$. However, one may also hypothesize that the photobionts confer a phenotypic impact on the lichen and that the abundant photobiont provides a fitness advantage of the individual thallus, (i.e., that 
a.

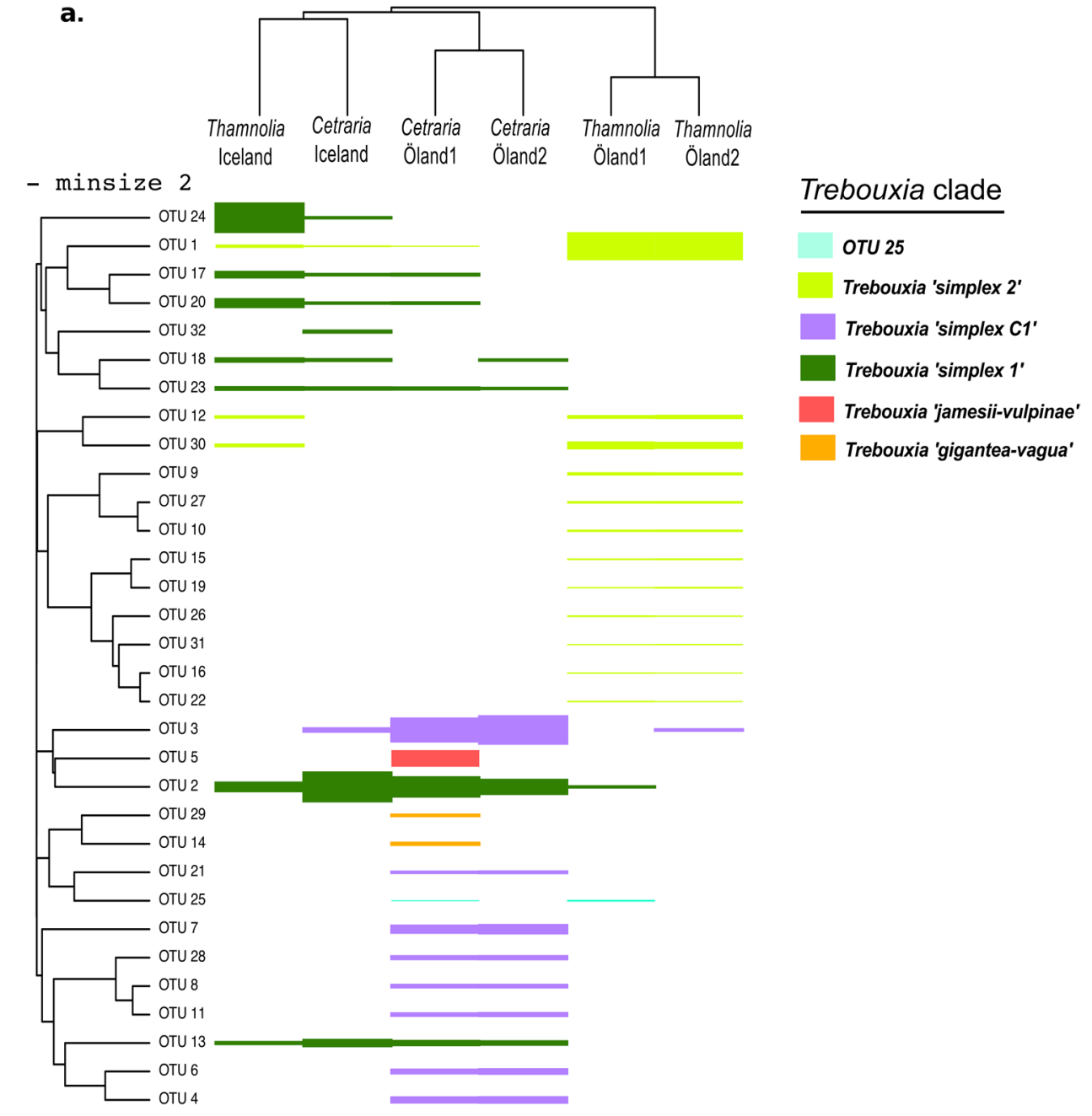

b.

Iceland

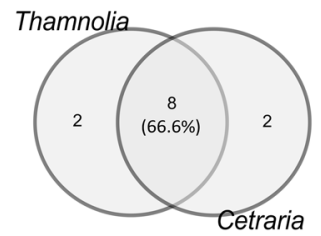

Öland 1

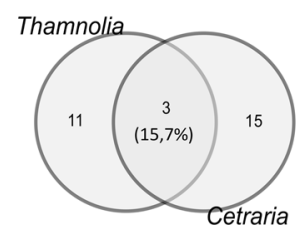

Öland 2

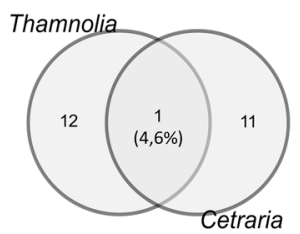

Figure 5. Photobiont diversity within the samples of Thamnolia and Cetraria, based on Ion Torrent data (a) Hierarchical clusters based on photobiont OTU relative quantities. The number of reads for each OTU in each sample is shown by bar thickness, which is scaled via its cube-root for display. The left dendrogram clusters OTUs by similarity in composition and abundances, and the top dendrogram clusters site/genus pairs by similarities in composition and abundances. The colors of the bars reflect the genotypic identity of each OTU based on the ITS1 photobiont phylogeny (Fig. 3). For all samples the reads were pooled, dereplicated, and clustered into OTUs using the constraint of requiring each OTU to be supported by at least two reads ( - minsize 2). (b) Venn diagrams showing the proportion of shared and exclusive OTUs between Thamnolia and Cetraria pooled samples within each locality.

the abundance of photobionts is driven by the adaptation to the local environment). Experiments investigating differential fitness of lichen thalli in response to relative abundance of photobionts have to our knowledge not been carried out. However, in line with this hypothesis, when photobiont lineage specific primers have been used in combination with monocultures of photobiont cells from lichen thalli, it has been shown that coexisting algal genotypes with complementary physiological performances ${ }^{15}$ could be found within a lichen individual ${ }^{33,69}$. Such selection of genetically similar but distinct photobionts within a thallus fits within the 'fungal-farmers hypothesis', in which mycobionts acquire available photobionts and subsequently select the appropriate genotype as 
the farming observed in ant growing fungi ${ }^{19}$ or the use of environmentally adapted photobionts in corals ${ }^{2,70}$. Yet another, not mutually exclusive, adaptive model is that the selection is acting on the photobiont, so that it increases in abundance in the thallus, but that this process is independent of the mycobiont.

The effect of lichen dispersal on photobiont community in thalli. Photobiont sharing between distantly related lichens suggest that horizontal transmission of photobiont occurs ${ }^{11}$ and our data supports this notion. However, the mechanism of photobiont recruitment in macrolichens such as Thamnolia and Cetraria with a predominantly or even exclusively vegetative dispersal mode is still not understood. Does photobiont switching take place only when fungal spores spread by themselves and resynthesize the lichen by acquiring a photobiont obtained from an external source? Independent dispersal of the symbionts to Iceland and local reassembling of the lichen in that area could explain some of the observed sharing of photobiont populations between Thamnolia and Cetraria and why Thamnolia specimens from Öland seem to contain a different spectrum of photobionts (Fig. 5a). Furthermore, our data of identical, or very similar, photobiont genotypes from different parts of the world (Fig. 3) suggest that the photobiont community is also affected by long-distance codispersal of the symbionts, which is likely to take place by vegetative propagules. One third factor that may affect the photobiont distribution in lichens is the continuous acquisition of photobionts after the lichen is formed, a phenomenon shown to take place in corals $^{70}$, but never proven in lichens. The combination of these routes of lichen dispersal and photobiont acquisition, together with the ability to maintain a diverse set of photobionts within a thallus, may be advantageous for exclusively or predominantly vegetative spreading lichen species to adapt to new environments.

\section{Conclusion}

Our results confirm that lichens may contain heterogeneous populations of photobionts that can be shared between species ${ }^{15,33-37}$. The lichen guild hypothesis postulates that distantly related but sympatric lichenized fungi may share a common photobiont pool ${ }^{21,24,34}$, and it was also shown that geographically separated lichens that occupy similar niches may have similar photobionts ${ }^{4,12,17,18}$. Based on this earlier work, together with our findings of similar genotypes across wide geographic ranges and the occurrence of multiple photobiont genotypes within Thamnolia and Cetraria thalli, we suggest an extended hypothesis regarding lichens adaption to the environment. We speculate that, just as reef-forming corals ${ }^{70}$, predominantly vegetatively dispersing lichens acquire photobionts during both incipient and adult life stages (idea also proposed in 2010 by Asplund and co-workers ${ }^{71}$ ). Depending on the environment, the most adapted photobiont increases in relative frequency within the thallus, at the same time as the accumulated intrathalline diversity is retained. Dispersal through vegetative propagules that maintain the photobiont diversity of the thallus will increase the chance for a successful establishment of lichen individuals in the new environments. Additional data from a diverse range of lichen species addressing for example the distribution of photobionts within individual thalli combined with fitness experiments, is needed in order to confirm this adaptive hypothesis and prove its generality in lichen symbiosis.

\section{References}

1. Douglas, A. E. The symbiotic habit. Princeton. (Princeton University Press., 2010).

2. Rowan, R. Coral bleaching: Thermal adaptation in reef coral symbionts. Nature 430, 742-742 (2004)

3. Poelt, J. In The Lichens (eds V. Ahmadjian \& M.E. Hale) 599-632 (Academic Press, 1973).

4. Fernandez-Mendoza, F. et al. Population structure of mycobionts and photobionts of the widespread lichen Cetraria aculeata. Mol. Ecol. 20, 1208-1232 (2011).

5. Sanders, W. B. \& Lücking, R. Reproductive strategies, relichenization and thallus development observed in situ in leaf-dwelling lichen communities. New Phytol. 155, 425-435 (2002).

6. Onuț-Brännström, I. The puzzle of lichen symbiosis: Pieces from Thamnolia 1503 thesis, Acta Universitatis Upsaliensis (2017).

7. Nash, H T. III. Lichen Biology. 2 edn, (ed. Nash, H, T. III), Cambridge University Press (2008).

8. Wornik, S. \& Grube, M. Joint dispersal does not imply maintenance of partnerships in lichen symbioses. Microb. Ecol. 59, 150-157 (2010).

9. Honegger, R. The Lichen Symbiosis - What is so spectacular about it? The Lichenologist 30, 193-212 (1998).

10. Beck, A. Photobiont inventory of a lichen community growing on heavy-metal-rich rock. The Lichenologist 31 (1999).

11. Piercey-Normore, M. D. \& DePriest, P. T. Algal Switching Among Lichen Symbiosis. Am. J. Bot. 88, 1490-1498 (2001).

12. Singh, G. et al. Fungal-algal association patterns in lichen symbiosis linked to macroclimate. New Phytologist 214, 317-329 (2017).

13. Piercey-Normore, M. D. \& Deduke, C. Fungal farmers or algal escorts: lichen adaptation from the algal perspective. Molec. Ecol. 20, 3708-3710 (2011).

14. Smith, E. G., Vaughan, G. O., Ketchum, R. N., McParland, D. \& Burt, J. A. Symbiont community stability through severe coral bleaching in a thermally extreme lagoon. Sci. Rep. 7, 2428 (2017).

15. Casano, L. M. et al. Two Trebouxia algae with different physiological performances are ever-present in lichen thalli of Ramalina farinacea. Coexistence versus competition? Environ. Microbiol. 13, 806-818 (2011).

16. Williams, L. et al. Lichen acclimation to changing environments: Photobiont switching vs. climate-specific uniqueness in Psora decipiens. Ecol. Evol 7, 2560-2574 (2017).

17. Peksa, O. \& Skaloud, P. Do photobionts influence the ecology of lichens? A case study of environmental preferences in symbiotic green alga Asterochloris (Trebouxiophyceae). Molec. Ecol. 20,3936-3948 (2011).

18. Werth, S. \& Sork, V. L. Ecological specialization in Trebouxia (Trebouxiophyceae) photobionts of Ramalina menziesii (Ramalinaceae) across six range-covering ecoregions of western North America. Am. J. Bot. 101, 1127-1140 (2014).

19. Lücking, R. et al. Do lichens domesticate photobionts like farmers domesticate crops? Evidence from a previously unrecognized lineage of filamentous cyanobacteria. Am. J. Bot. 96, 1409-1418 (2009).

20. Perez-Ortega, S., Ortiz-Alvarez, R., Allan Green, T. G. \& de Los Rios, A. Lichen myco- and photobiont diversity and their relationships at the edge of life (McMurdo Dry Valleys, Antarctica). FEMS Microbiol. Ecol. 82, 429-448 (2012).

21. Rikkinen, J., Oksanen, I. \& Lohtander, K. Lichen Guilds Share Related Cyanobacterial Symbionts. Science 297, 357 (2002).

22. Beck, A., Friedl, T. \& Rambold, G. Selectivity of photobiont choice in a defined lichen community: inferences from cultural and molecular studies. New Phytol. 139, 709-720 (1998).

23. Belinchón, R., Yahr, R. \& Ellis, C. J. Interactions among species with contrasting dispersal modes explain distributions for epiphytic lichens. Ecography 38, 762-768 (2015). 
24. Dal Grande, F. et al. Molecular phylogeny and symbiotic selectivity of the green algal genus Dictyochloropsis s.l. (Trebouxiophyceae): a polyphyletic and widespread group forming photobiont-mediated guilds in the lichen family. Lobariaceae. New Phytol. 202, 455-470 (2014).

25. Engelen, A., Convey, P., Popa, O. \& Ott, S. Lichen photobiont diversity and selectivity at the southern limit of the maritime Antarctic region (Coal Nunatak, Alexander Island). Polar Biol (2016).

26. Hestmark, G., Lutzoni, F. \& Miadlikowska, J. Photobiont associations in co-occurring umbilicate lichens with contrasting modes of reproduction in coastal Norway. The Lichenologist 48, 545-557 (2016).

27. Leavitt, S. D. et al. Fungal specificity and selectivity for algae play a major role in determining lichen partnerships across diverse ecogeographic regions in the lichen-forming family Parmeliaceae (Ascomycota). Molec. Ecol. 24, 3779-3797 (2015).

28. Ruprecht, U., Brunauer, G. \& Printzen, C. Genetic diversity of photobionts in Antarctic lecideoid lichens from an ecological view point. The Lichenologist $\mathbf{4 4}$ (2012).

29. Vargas Castillo, R. \& Beck, A. Photobiont selectivity and specificity in Caloplaca species in a fog-induced community in the Atacama Desert, northern Chile. Fungal Biol. 116, 665-676 (2012).

30. Wirtz, N. et al. Lichen fungi have low cyanobiont selectivity in maritime Antarctica. New Phytol. 160, 177-183 (2003).

31. Onuţ-Brännström, I., Tibell, L. \& Johannesson, H. A worldwide phylogeography of the whiteworm lichens Thamnolia reveals three lineages with distinct habitats and evolutionary histories. Ecol. and Evol. 7 (2017).

32. Cao, S. et al. Distribution patterns of haplotypes for symbionts from Umbilicaria esculenta and U. muehlenbergii reflect the importance of reproductive strategy in shaping population genetic structure. BMC Microbiol. 15, 212 (2015).

33. Beck, A., Kasalicky, T. \& Rambold, G. Myco-photobiontal selection in a Mediterranean cryptogam community with Fulgensia fulgida. New Phytol. 153, 317-326 (2002).

34. Dal Grande, F. et al. Insights into intrathalline genetic diversity of the cosmopolitan lichen symbiotic green alga Trebouxia decolorans Ahmadjian using microsatellite markers. Mol. Phylogenet. Evol. 72, 54-60 (2014).

35. Dal Grande, F. et al. Environment and host identity structure communities of green algal symbionts in lichens. New Phytol, 277-289 (2017).

36. Moya, P., Molins, A., Martinez-Alberola, F., Muggia, L. \& Barreno, E. Unexpected associated microalgal diversity in the lichen Ramalina farinacea is uncovered by pyrosequencing analyses. PloS One 12 (2017).

37. Piercey-Normore, M. D. The lichen-forming ascomycete Evernia mesomorpha associates with multiple genotypes of Trebouxia jamesii. New Phytol 169, 331-344 (2006).

38. Miadlikowska, J. et al. A multigene phylogenetic synthesis for the class Lecanoromycetes (Ascomycota): 1307 fungi representing 1139 infrageneric taxa, 317 genera and 66 families. Mol. Phylogenet. Evol 79, 132-168 (2014).

39. Nelsen, M. P. \& Gargas, A. Symbiont flexibility in Thamnolia vermicularis (Pertusariales: Icmadophilaceae). Bryologist 112, 404-417 (2009).

40. Andrei, M., Iacob, M. \& Pascale, M. Vegetative multiplication in Thamnolia vermicularis (Sw.) Schaer. Romanian Journal of Biology. - Plant Biol. 51-52, 55-57 (2006-2007).

41. Nelsen, M. P. \& Gargas, A. Assessing clonality and chemotype monophyly in Thamnolia (Icmadophilaceae). Bryologist 112, 42-53 (2009).

42. Cassie, D. M. \& Piercey-Normore, M. D. Dispersal in a sterile lichen-forming fungus. Thamnolia subuliformis (Ascomycotina: Icmadophilaceae). Botany 86, 751-762 (2008).

43. Lord, J. M. et al. Rediscovery of pycnidia in Thamnolia vermicularis: implications for chemotype occurrence and distribution. Lichenologist 45, 397-411 (2013).

44. Venzke, J. F. On the Ecology and plant-sociology of "melur" - vegetation in Iceland. Acta-Botanica-Islandica 9, 3-18 (1987).

45. Rosén, E. \& van der Maarel, E. Restoration of alvar vegetation on Öland, Sweden. Appl. Veg. Sci. 3, 65-72 (2000).

46. Barron, V. \& Torrent, J. Use of the Kubelka-Munk theory to study the influence of iron oxides on soil colour. Eur. J. Soil Sci. 37, 499-510 (1986)

47. Kearse, M. et al. Geneious Basic: an integrated and extendable desktop software platform for the organization and analysis of sequence data. Bioinformatics 28, 1647-1649 (2012).

48. Edgar, R. C. Search and clustering orders of magnitude faster than BLAST. Bioinformatics 26, 2460-2461 (2010).

49. Caporaso, J. G. et al. QIIME allows analysis of high-throughput community sequencing data. Nat. Meth. 7, 335-336 (2010).

50. Colwell, R. K. et al. Models and estimators linking individual-based and sample-based rarefaction, extrapolation and comparison of assemblages. J. Plant Ecol. 5 (2012).

51. Benson, D. A., Karsch-Mizrachi, I., Lipman, D. J., Ostell, J. \& Wheeler, D. L. GenBank. Nucleic Acids Res. 33 (2005)

52. Katoh, S. MAFFT multiple sequence alignment software version 7: improvements in performance and usability. (outlines version 7). Mol. Biol. Evol. 30, 772-780 (2013).

53. Larsson, A. AliView: a fast and lightweight alignment viewer and editor for large data sets. Bioinformatics 30, 3276-3278 (2014).

54. Lischer, H. E., Excoffier, L. \& Heckel, G. Ignoring heterozygous sites biases phylogenomic estimates of divergence times: implications for the evolutionary history of microtus voles. Mol. Biol. Evol. 31, 817-831 (2014).

55. Bazinet, A. L., Zwicki, D. J. \& Cummings, M. P. A Gateway for Phylogenetic Analysis Powered by Grid Computing Featuring GARLI 2.0. Syst. Biol. 63, 812-818 (2014).

56. Sukumaran, J. \& Holder, M. T. DendroPy: a Python library for phylogenetic computing. Bioinformatics 26, 1569-1571 (2010).

57. Redelings, B. Erasing errors due to alignment ambiguity when estimating positive selection. Mol. Biol. Evol 31, 1979-1993 (2014).

58. Darriba, D., Taboada, G., Doallo, R. \& Posada, D. jModelTest 2: more models, new heuristics and parallel computing. Nat. Meth. 9(8), 772 (2012)

59. Rambaut A, Suchard MA, Xie D \& Drummond AJ Tracer v1.6, Available from http://tree.bio.ed.ac.uk/software/tracer/ (2014).

60. Oksanen, J. et al. Vegan: Community Ecology Package. R package version 2.4-0. http://CRAN.R-project.org/package=vegan (2016).

61. Wickham, H. ggplot2: Elegant Graphics for Data Analysis. Springer-Verlag, New York (2009).

62. Onut-Brännström, I., Johannesson, H. \& Tibell, L. Thamnolia tundrae n. sp., a cryptic species and putative glacial relict. The Lichenologist 50-1, 59-75 (2018).

63. Muggia, L. et al. The symbiotic playground of lichen thalli-a highly flexible photobiont association in rock-inhabiting lichens. FEMS Microbiol. Ecol 85, 313-323 (2013).

64. Spribille T. et al. Basidiomycete yeasts in the cortex of ascomycete macrolichens. Science 353(6298), 488-492 (2016).

65. LaJeunesse, T. C. et al. Long-standing environmental conditions, geographic isolation and host-symbiont specificity influence the relative ecological dominance and genetic diversification of coral endosymbionts in the genus. Symbiodinium. J. Biogeogr. 37, 785-800 (2010)

66. Park, C. H. et al. Algal and fungal diversity in Antarctic lichens. J Eukaryot Microbiol 62, 196-205 (2015).

67. Lutsak, T., Fernández-Mendoza, F., Kirika, P., Wondafrash, M. \& Printzen, C. Mycobiont-photobiont interactions of the lichen Cetraria aculeata in high alpine regions of East Africa and South America. Symbiosis 68, 25-37 (2015).

68. Magain, N., Miadlikowska, J., Goffinet, B., Serusiaux, E. \& Lutzoni, F. Macroevolution of Specificity in Cyanolichens of the Genus Peltigera Section Polydactylon (Lecanoromycetes, Ascomycota). Syst. Biol. 66, 74-99 (2017).

69. Catala, S. et al. Coordinated ultrastructural and phylogenomic analyses shed light on the hidden phycobiont diversity of Trebouxia microalgae in Ramalina fraxinea. Mol. Phylogenet. Evol 94, 765-777 (2016). 
70. Andras, J. P., Rypien, K. L. \& Harvell, C. D. Range-wide population genetic structure of the Caribbean sea fan coral. Gorgonia ventalina. Molec. Ecol. 22, 56-73 (2013).

71. Asplund, J., Larsson, P., Vatne, S. \& Gauslaa, Y. Gastropod grazing shapes the vertical distribution of epiphytic lichens in forest canopies. J. of Ecol. 98, 218-225 (2010).

\section{Acknowledgements}

We would like to thank Petter Madsen for testing for the existence of multiple photobionts in Thamnolia lichen thalli with specific primers. We also like to thank Diem Nguyen for fruitful discussions. Computations were performed on resources provided by SciLifeLab (www.scilifelab.se) and the Swedish National Infrastructure for Computing (http://www.snic.vr.se/) through UPPMAX (www.uppmax.uu.se) under projects b2013277. This work was supported by grants from Kungliga Vetenskapsakademien, the Helge Ax:Son Johnsons, Lars Hiertas Minne and Nilsson-Ehle Foundations as well as several Uppsala University scholarships: Regnells botaniska resetipendium, Linne travel scholarship and Tullbergs stipendium for biological research.

\section{Author Contributions}

I.O.B., M.B., D.G.S. and H.J. planned and designed the research, I.O.B. and S.H. performed the collection; M.B. and I.O.B performed the lab work; D.G.S., I.O.B., M.G.I.A. and E.S.L. performed the data analyses; I.O.B., M.B., D.G.S., M.G.I.A., E.S.L. and H.J. interpreted the results and wrote the manuscript.

\section{Additional Information}

Supplementary information accompanies this paper at https://doi.org/10.1038/s41598-018-22470-y.

Competing Interests: The authors declare no competing interests.

Publisher's note: Springer Nature remains neutral with regard to jurisdictional claims in published maps and institutional affiliations.

(c) (i) Open Access This article is licensed under a Creative Commons Attribution 4.0 International License, which permits use, sharing, adaptation, distribution and reproduction in any medium or format, as long as you give appropriate credit to the original author(s) and the source, provide a link to the Creative Commons license, and indicate if changes were made. The images or other third party material in this article are included in the article's Creative Commons license, unless indicated otherwise in a credit line to the material. If material is not included in the article's Creative Commons license and your intended use is not permitted by statutory regulation or exceeds the permitted use, you will need to obtain permission directly from the copyright holder. To view a copy of this license, visit http://creativecommons.org/licenses/by/4.0/.

(c) The Author(s) 2018 